\title{
Time Stamp with Authentication Protocol for Securing Tag Reader Communication
}

\author{
Harshad Panwar \\ M.Tech(CS) \\ Govt. Engg. College \\ Ajmer
}

\author{
Umesh Kumar Garg \\ M.Tech(CS) \\ Govt. Engg. College \\ Ajmer
}

\author{
Neetu Sharma \\ Asst. Professor \\ Govt. Engg. College \\ Ajmer
}

\begin{abstract}
RFID is evolving as a major technology. Since 1940s its use in different domains shows its gaining importance also it's a better alternative to barcode as no line of sight is required. Here we have showed the use of time stamp that validates the authenticity of the sender to be legal or authorized. So, copying of tag is restricted by a large [3]. The proposed plan is to prevent the RFID system form attack by foreign agent. At the time of connection initiation when the two entities are authenticated and secondly to assure that the connection is not breached. Here, hash function and time stamp are used in conjunction with message [6]. This is a scalable and efficient mechanism to check eavesdropper illegal entry into the communication channel.
\end{abstract}

\section{General Terms}

Security algorithm, Forward security.

\section{Keywords}

RFID Authentication, Time stamp, Replay attack.

\section{INTRODUCTION}

Rfid (Radio frequency identification) is used in numerous applications that includes it is industries or in mega stores, in tracking vehicles in a city, and recently passports will be equipped with RFID device to speed border crossings at busy airports etc. Rfid system has three basic components a tag (wireless with an antenna), a reader and a back-end server. The tag and reader works on the principle of NFC (near field communication).

Therefore, the communication channel between the tag and the reader is prone to invasion and requires security, for which use of hash based function in conjunction with time stamp has been proposed [1]. The communication is initiated by reader with a unique tag or set of tags. In this process there are chances that an adversary can breach by replay attack.

So, the proposed paper is an attempt to provide a concrete feature to rampart by generating the hash code of the message and sending time stamp along with it by the reader to the tag. So that if contravention is carried out, can be prohibited, and replay attack is averted.

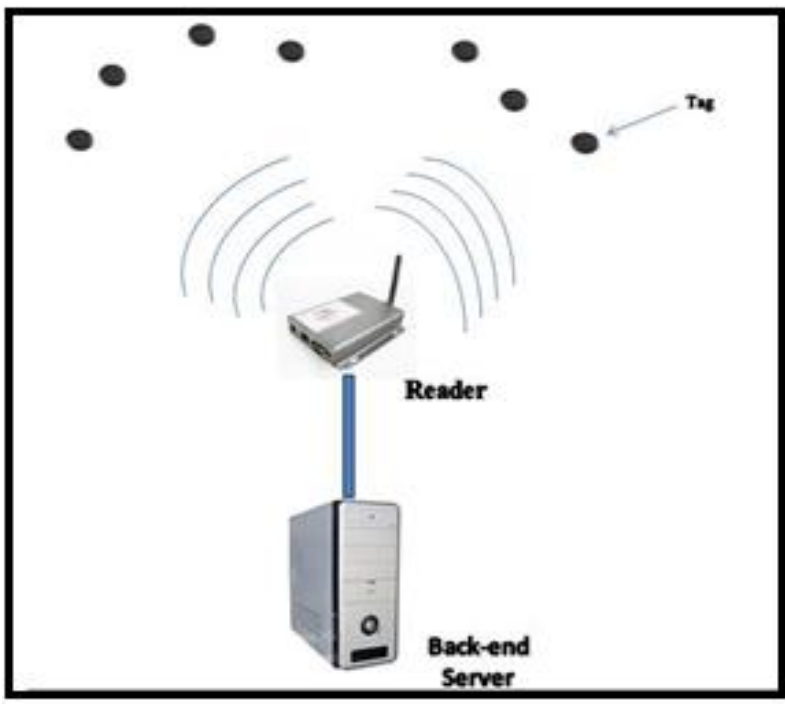

Fig.1: Tag - Reader Communication with backend Server

\section{PROPOSED PROTOCOL}

In the proposed plan we are trying to update the key shared by the tag \& reader and PIN shared by the Tag and Back-end Server. This protocol works on hash function so that no one can convert the original form of the message after hashing. And we use time stamp so that the system can be prevented from Replay attack [4]. And update the value of key and PIN so that if adversary is able to find the key and PIN, after that this key is of no use for adversary. The proposed authentication protocol is shown in fig 2 .

Step 1: Reader first generates a fresh random time being $\mathrm{K}$, randomizes it with the one-way hash function, $\mathrm{C} 1=\mathrm{H}(\mathrm{K})$ followed by checking time stamp from the time stamp generator and sends time stamp along with hash function to the tag $\mathrm{T}$.

Step 2: When Tag receives the message, first it compares the time stamp with previous time stamp value stored in its nonvolatile memory [2]. If previous time stamp value is less than the received value, it proceeds to the hashing code. Tag compares the message with the generated hash value. If both values are equal then tag assures that the received message is authentic, and updates its time stamp with received time stamp. 


\begin{tabular}{|c|c|c|}
\hline Tag T & Reader $\mathrm{R}$ & Back-end Server S \\
\hline $\mathrm{K} I \mathrm{D}_{\mathrm{i}} \mathrm{PIN}, \mathrm{TSP}_{\text {last }}$ & $\mathrm{K}$ & $\begin{array}{l}E P C, H\left(I D_{j}\right), I D_{j}, P I N, H\left(I_{\text {lat }}\right) \text {, } \\
I_{\text {lat, }}, P \mid N_{\text {last }}\end{array}$ \\
\hline & $\begin{array}{l}\text { 1. Gets TSP } \\
\mathrm{C} 1=\mathrm{H}(\mathrm{K}) \mathrm{TSP}\end{array}$ & \\
\hline \multicolumn{3}{|c|}{$\mathrm{C} 1, \mathrm{TSP}$} \\
\hline \multicolumn{3}{|l|}{$\begin{array}{l}\text { 2. If TSP>TSP } \\
\text { Then } \\
C 2=H(K, T S P) \\
\text { If } C 1==C 2 \\
\text { Then send } H\left(I D_{j}\right) \\
\text { And Update TSP by } \\
\text { TSP last }=T S P\end{array}$} \\
\hline \multicolumn{3}{|c|}{ 3. $\stackrel{\mathrm{H}\left(\mathrm{ID} \mathrm{D}_{\mathrm{j}}\right)}{\longrightarrow}$} \\
\hline & & $\begin{array}{l}\text { Finds ID, } \\
\text { Update hash and ID value } \\
C 3=H\left(D_{i} \text { PIN }\right) \\
I_{i+1}=H\left(D_{i} \text { PIN }, T S P\right) \\
P_{\text {last }}=\text { PIN } \\
P I N=H(P I N, T S P, C 3)\end{array}$ \\
\hline \multicolumn{3}{|c|}{ 5. C3 } \\
\hline & 6. $\mathrm{K}=\mathrm{H}(\mathrm{K}, \mathrm{TSP})$ & \\
\hline \multicolumn{3}{|c|}{ 7. $\mathrm{C} 3, \mathrm{TSP}$} \\
\hline 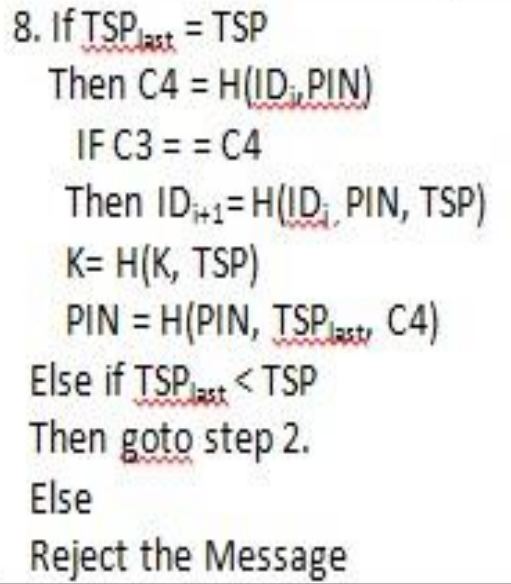 & & \\
\hline
\end{tabular}

Fig.2: The Proposed Authentication protocol 
Step 3: Tag sends its pseudo ID with hashing to the reader.

Step 4: Reader simply forwards this message as it is to the Back-end Server.

Step 5: After receiving the message Back-end server determines the $\mathrm{H}$ (ID) from its data base and if it does not found then find in the $\mathrm{ID}_{\text {last }}$. And extract the PIN and generate the hashing value with ID and PIN and update the value of ID and update the value of PIN. Send the message to the reader.

Step 6: Reader updates its key value by hash function.

Step 7: Reader sends the message to the tag with time stamp.

Step 8: After receiving the message, Tag compares the time stamp with previous time stamp [5]. If both are equal then Tag calculates the message with hashing of ID with Pin and compares this against received message. Values are equal then tag authenticates the back-end server and the authentication has been successful and after that it updates its value of ID, Key and PIN. Otherwise reject the message.

\section{ANALYSIS}

\subsection{Synchronization}

The simplified TD protocol happens to desynchronization problem. TD protects desynchronization between $\mathrm{S}$ and tags at the last step in enhanced TD protocol. In our protocol desynchronization can occur only if $\mathrm{T}$ updates its ID and $\mathrm{S}$ does not (this will happen if message 5 is lost or corrupted). However as the S maintains two lists of ID's, so it can easily finds out ID from ID $_{\text {last }}$ list and thus avoids desynchronization.

\subsection{Forward Secrecy}

Our protocol updates IDi to IDi+1 using a one-way hash function $\mathrm{h}$ () like OSK and TD. As long as there is no alternative, we have to use one way function to guarantee forward secrecy.

\subsection{Un-traceability}

Tags authenticate $\mathrm{R}$ after receiving the first message; $\mathrm{R}$ authenticates the tags after receiving the second message. In each step, tags and $\mathrm{R}$ authenticate counterpart to remove traceability. In addition, although adversary $\boldsymbol{A}$ knows $\mathrm{k}$, A cannot trace a particular $\mathrm{T}$ since Ts response to query is always different at the valid session.

\section{CONCLUSION}

The earlier designed hash lock schemes are scalable, but traceable and randomized hash lock schemes are untraceable, but un-scalable [9]. Therefore, we designed a scalable and untraceable protocol in order to provide data integrity and security. Our protocol is more secure than TD in terms of traceability aspects even though ours reduces hash operations from five and more to four. In our protocol, $\mathrm{T}$ needs four hash operations to communicate with $\mathrm{R}$ with quite good security performance [8]. Under the assumption that tags cannot be tampered, we don't need to send even the last message. The protocol supports ownership transfer using k. As far as we know, ownership transfer issue is dealt with rarely so far. For example, Alice has $\mathrm{R}$ that has $\mathrm{k}$ which is also stored in tagged items of Alice. When Alice gets some tagged items from Bob, Alice can write her own k.

\section{REFRENCES}

[1] National Institute of Standards and Technology "Guidelines for securing Radio Frequency Identification (RFID) systems" NIST special publication 800-98.

[2] Sindhu Karthikeyan and Mikhail Nesterenko "RFID Security without Extensive Cryptography" SASN'05, November 7, 2005, Alexandria, Virginia, USA.

[3] Gildas Avoine and Philippe Oechslin, "A Scalable and Provably Secure Hash based RFID Protocol", In International Workshop on Pervasive Computing and Communication Security - PerSec 2005, pp.110-114, Mar. 2005, IEEE Computer Society Press, Kauai Island, Hawaii, USA.

[4] Gene Tsudik, "YA-TRAP: Yet Another Trivial RFID Authentication Protocol", International Conference on Pervasive Computing and Communications PerCom 2006, Mar. 2006, IEEE Computer Society Press, Pisa, Italy.

[5] Istv'an Vajda and Levente Butty'an, "lightweight Authentication Protocol for Low-Cost RFID Tags", International Conference on Computational Science and its Applications - ICCSA 2005, LNCS 3480, pp.619-627, May 2005, Springer-Verlag, Singapore.

[6] Miyako Ohkubo, Koutarou Suzuki and Shingo Kinoshita, "Cryptographic Approach to Privacyfriendly Tags", In RFID Privacy Workshop, 2003, MIT, USA.

[7] Stephen Weis, Sanjay Sharma, Ronald Rivest and Daniel Engels, "Security and Privacy Aspects of Low-Cost Radio FrequencyIdentification Systems", Conference on Security in Pervasive Computing SPC 2003, LNCS 2802, pp.454-469, Mar. 2003, Springer-Verlag, Boppard, Germany.

[8] Tassos Dimitriou, "A Lightweight RFID Protocol to protect against Traceability and Cloning attacks", Conference on Security andPrivacy for Emerging Areas in Communication Networks SecureComm'05, pp.59-66, Sep. 2005, Athens, Greece.

[9] Jeongkyu Yang, Jaemin Park, Hyunrok Lee, Kui Ren and Kwangjo 